\title{
THE GOMPERTZ FUNCTION AND ITS APPLICATIONS IN MANAGEMENT
}

\author{
Grzegorz RZĄDKOWSKI*, Iwona GŁAŻEWSKA**, Katarzyna SAWIŃSKA*** \\ Faculty of Management, Warsaw University of Technology, Warsaw, Poland \\ *e-mail: g.rzadkowski@wz.pw.edu.pl \\ **e-mail: i.glazewska@wz.pw.edu.pl \\ ***e-mail: katarzyna-s@o2.pl
}

\begin{abstract}
In the present paper, we investigate the Gompertz function, which is commonly used, mostly as diffusion model, in economics and management. Our approach is based on indicating in a given time series, presumably with a Gompertz trend, some characteristic points corresponding to zeroes of successive derivatives of this function. This allows us to predict the saturation level of a phenomenon under investigation, by using only the early values of the time series. We also give an example of applications of this method.
\end{abstract}

Keywords: Gompertz function, time series, Stirling number of the II kind, mathematical model.

\section{Introduction}

The Gompertz function is described by the following differential equation of the first order

$$
u^{\prime}(t)=q u \log \frac{u_{\max }}{u}, \quad u(0)=u_{0}>0,
$$

where $\mathrm{t}$ denotes time (or expenditures), $\mathrm{u}=\mathrm{u}(\mathrm{t})$ is the unknown function (a solution of equation (1)), $\mathrm{q}, \mathrm{u}_{\max }$ are constants (parameters of the equation) and $\log (\mathrm{x})$ stands for the natural logarithm. The constant $u_{\max }$ is called a saturation level. The integral curve $u=u(t)$ of the equation (1), fulfilling condition $0<\mathrm{u}(\mathrm{t})<\mathrm{u}_{\max }$ is known as the Gompertz function.

A phenomenon described by equation (1) and function $u(t)$ has an important property that its rate of growth $\mathrm{u}^{\prime}(\mathrm{t})$ is proportional to the level already achieved i.e., $u(t)$. On the other hand, if $u(t)$ is sufficiently large and close to $\mathrm{u}_{\max }$, then the factor $\log \frac{\mathrm{u}_{\max }}{\mathrm{u}}$ is more significant and its influence inhibits further growth of the function $u(t)$.

From mathematical point of view equation (1) is the first order ordinary differential equation, which is easily solved by the method of the separation of variables. After solving it we obtain the following formula for the Gompertz function

$$
\mathrm{u}(\mathrm{t})=\mathrm{u}_{\max } \mathrm{e}^{-\mathrm{ce} \mathrm{e}^{-\mathrm{t}}},
$$

where constant $c$ appears in the integration process of (1) and is connected with the initial condition $\mathrm{u}(0)=\mathrm{u}_{0}=\mathrm{u}_{\max } \mathrm{e}^{-\mathrm{c}}$, thus $\mathrm{c}=\log \frac{\mathrm{u}_{\max }}{\mathrm{u}_{0}}>0$.

Mahjan et al. [4] show areas in which various S-shaped curves are used as diffusion models. The authors find that the Bass function can be used for modeling of consumer durable goods, retail services, agricultural, education, and industrial innovations. The logistic curve serves as model in industrial, high technology, administrative innovations, and the Gompertz function can be used for modeling consumer durable goods and agriculture innovations. This division into the areas of application is then confirmed in other studies (see [2, and 3]). Many different applications of the Gompertz curve are given moreover in papers [7, and 8].

The main idea of the present paper is to look, among the data of a given time series, for some characteristic points, which correspond to zeroes of derivatives of the Gompertz function. One of these points is clearly the point corresponding to the inflection point (i.e., the zero of $\mathrm{u}^{\prime \prime}$ ) of the Gompertz curve, at which, as is well known, the function takes value $0,368 \mathrm{u}_{\max }$.

For a sufficiently long time series, the point corresponding to the inflection point is easy to locate approximately, even from the graph. 
If the data were collected for the times spaced equally, then, instead of estimating the values of the first derivative, it is sufficient to calculate successive first order differences and seek for their maximum to find an approximate value of the inflection point.

What we can do however, when the time series is not long enough, and we expect that the investigated phenomenon follows the Gompertz curve? When the phenomenon is on an early stage of growth and the data is available only in a relatively short time interval? Statistical methods for estimating the parameters of the Gompertz function based, for example, on the method of the nonlinear least squares may be unreliable, since functions having significantly different values of the saturation level may produce only slightly differing error values.

A way to explain of the situation seems in seeking, in the time series, points corresponding to zeroes of successive derivatives of the Gompertz function. For equally spaced time points this is equivalent to calculating successive differences.

For example, as we will see in Section 3, the zero of the third derivative $u^{\prime \prime \prime}$ (i.e., the extreme (maximum) of the second derivative $\mathrm{u}^{\prime \prime}$ ) occurs at the point, where the value of the logistic function is approximately $0,0729 \mathrm{u}_{\max }$.

Similar method was used in our paper [5] for phenomena having the logistic trend.

By considering examples from the field of economics and management we have demonstrated its usefulness and effectiveness.

In the present article, we use some formulas concerning the Gompertz function, which have been proved in paper [6].

\section{Properties of the Gompertz function}

In contrast to the logistic function the Gompertz function does not have a symmetrical first derivative. Therefore, at the inflection point, the Gompertz function takes approximately the value $0,368 \mathrm{u}_{\text {max }}$, which is less than $u_{\max } / 2$ (it is the value of the logistic function at its inflection point).

The graph of an exemplary Gompertz function is shown in Fig. 1.

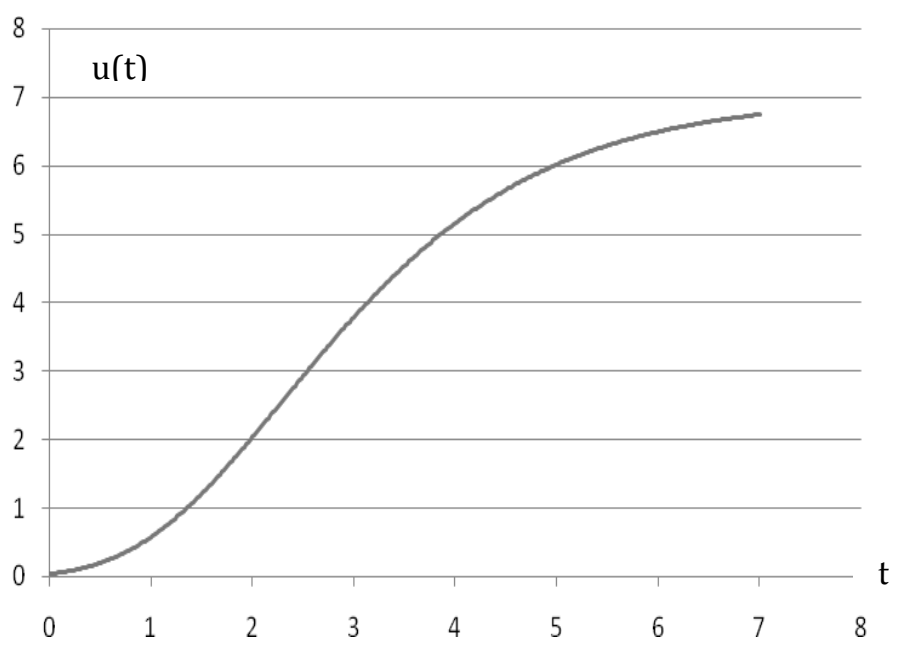

Figure 1. The Gompertz function with parameters $\mathrm{q}=0,7 ; \mathrm{u}_{\max }=7 ; \mathrm{c}=5$

(source: own elaboration in a spreadsheet)

We will introduce now Stirling numbers of the II kind because they are used in formulas for higher derivatives of the Gompertz function (see [1] for a good description of these numbers).
The Stirling number of the II kind, denoted by Knuth by $\left\{\begin{array}{l}n \\ k\end{array}\right\}$ is defined in combinatorics as the number of ways of partitioning a set of $\mathrm{n}$ elements into $\mathrm{k}$ nonempty subsets. 
For example three elements set consisting, say of numbers 1, 2, 3 can be partitioned into two nonempty subsets in three ways $(\{1\}$ and $\{2,3\}$ or $\{2\}$ and $\{1,3\}$ or $\{3\}$ and $\{1,2\})$, only one way into three nonempty subsets $(\{1\},\{2\}$ and $\{3\})$ and also one way into one nonempty subset (in this case it is the whole set $\{1,2,3\}$ ).

Table 1. Stirling numbers of the II kind (source: Graham, Knuth and Patashnik [1])

\begin{tabular}{|c|c|c|c|c|c|c|c|}
\hline $\mathrm{n}$ & $\left\{\begin{array}{l}\mathrm{n} \\
1\end{array}\right\}$ & $\left\{\begin{array}{l}\mathrm{n} \\
2\end{array}\right\}$ & $\left\{\begin{array}{l}\mathrm{n} \\
3\end{array}\right\}$ & $\left\{\begin{array}{l}\mathrm{n} \\
4\end{array}\right\}$ & $\left\{\begin{array}{l}\mathrm{n} \\
5\end{array}\right\}$ & $\left\{\begin{array}{l}n \\
6\end{array}\right\}$ \\
\hline 1 & 1 & & & & & \\
\hline 2 & 1 & 1 & & & & & \\
\hline 3 & 1 & 3 & 1 & & & & \\
\hline 4 & 1 & 7 & 6 & 1 & & & \\
\hline 5 & 1 & 15 & 25 & 10 & 1 & & \\
\hline 6 & 1 & 31 & 90 & 65 & 15 & 1 & \\
\hline 7 & 1 & 63 & 301 & 350 & 140 & 21 & 1 \\
\hline
\end{tabular}

The Stirling numbers of the II kind, as well as Newton binomial numbers, in addition to use in combinatorics, appear in many formulas of the broadly understood mathematical analysis.

$$
u^{(n)}(t)=q^{n} \sum_{k=1}^{n}(-1)^{n-k}\left\{\begin{array}{l}
n \\
k
\end{array}\right\} u \log ^{k} \frac{u_{\max }}{u}
$$

Using formula (3) and Table 1 let us write down formulas for the first few derivatives

$$
\begin{gathered}
u^{\prime \prime}(t)=q^{2} u \log \frac{u_{\max }}{u}\left(-1+\log \frac{u_{\max }}{u}\right) \\
u^{\prime \prime \prime}(t)=q^{3} u \log \frac{u_{\max }}{u}\left(1-3 \log \frac{u_{\max }}{u}+\log ^{2} \frac{u_{\max }}{u}\right) \\
u^{(4)}(t)=q^{4} u \log \frac{u_{\max }}{u}\left(-1+7 \log \frac{u_{\max }}{u}-6 \log ^{2} \frac{u_{\max }}{u}+\log ^{3} \frac{u_{\max }}{u}\right)
\end{gathered}
$$

From formula (4) it follows, what we have already mentioned, that the value of the Gompertz function at the only inflection point is equal:

$\mathrm{u}_{\max } / \mathrm{e} \approx 0,368 \mathrm{u}_{\max }$.

In turn, by analyzing the quadratic function defined in brackets on the right hand side of (5), we easily come to the conclusion that the value of the Gompertz function at the least zero point of its third derivative (it is simultaneously the maximum point of the second derivative) is equal:

$$
\exp (-(3+\sqrt{5}) / 2) \mathrm{u}_{\max } \approx 0,0729 \mathrm{u}_{\max } \text {. }
$$


Similarly, numerically computing the zeroes of the cubic polynomial in the brackets of (6) (note that the Cardano formulas give exact expressions but they are too difficult for practical use in this case) we are able to estimate the value of the Gompertz function at the least zero point of its fourth derivative as $\exp (-4,49086) \mathrm{u}_{\max } \approx 0,0112 \mathrm{u}_{\max }$.

\section{Applications}

We will now attempt to apply the results derived in the previous section for actual data. The data are presented in Table 2 in column 2 and are for sale in the consecutive 41 weeks in year 2013 (Week, column 1) of bicycle accessories (expressed in hundreds PLN rounded to the nearest hundred after completing the calculation) in a large retail chain of sport goods in Poland. The name of the retail chain has been reserved. column 3 shows the Total Sale, cumulatively in weeks 1 to 41 (see also Fig. 2). column 4 contains the exponentially smoothed values from column 2, with a smoothing constant equal to 0.17 , and column 5 consecutive sums of the numbers from column 4 . We will try to predict the saturation level using only the early part of the time series.

Table 2. Working table

(source: own elaboration based on the collected data)

\begin{tabular}{|c|c|c|c|c|c|c|}
\hline $\begin{array}{c}\text { Week } \\
-1-\end{array}$ & $\begin{array}{c}\text { Sales } \\
-2-\end{array}$ & $\begin{array}{c}\text { Total Sales } \\
-3-\end{array}$ & $\begin{array}{c}\text { Exponential } \\
\text { smoothing } \\
-4-\end{array}$ & $\begin{array}{c}\text { Total } \\
-5-\end{array}$ & $\begin{array}{l}\Delta^{2} y \\
-6-\end{array}$ & $\begin{array}{l}\Delta^{3} \mathrm{y} \\
-7-\end{array}$ \\
\hline 1 & 5117 & 5117 & 5117 & 5117 & & \\
\hline 2 & 5569 & 10686 & 5117 & 10233 & & \\
\hline 3 & 5896 & 16582 & 5194 & 15427 & 77 & \\
\hline 4 & 4929 & 21511 & 5313 & 20740 & 119 & 43 \\
\hline 5 & 8110 & 29621 & 5248 & 25988 & 65 & 185 \\
\hline 6 & 8491 & 38112 & 5734 & 31722 & 487 & 552 \\
\hline 7 & 9265 & 47376 & 6203 & 37925 & 469 & 18 \\
\hline 8 & 12028 & 59405 & 6723 & 44648 & 521 & 52 \\
\hline 9 & 44264 & 103669 & 7625 & 52274 & 902 & 381 \\
\hline 10 & 67592 & 171262 & 13854 & 66128 & 6229 & 5327 \\
\hline 11 & 53566 & 224828 & 22989 & 89117 & 9136 & 2907 \\
\hline 12 & 48381 & 273209 & 28188 & 117305 & 5198 & -3937 \\
\hline 13 & 51245 & 324454 & 31620 & 148925 & 3433 & -1765 \\
\hline 14 & 84510 & 408964 & 34957 & 183882 & 3336 & - \\
\hline 15 & 200786 & 609750 & 43381 & 227262 & 8424 & 5088 \\
\hline 16 & 326679 & 936429 & 70140 & 297402 & 26759 & 18335 \\
\hline 17 & 359837 & 1296266 & 113751 & 411153 & 43612 & 16853 \\
\hline 18 & 286480 & 1582747 & 155586 & 566739 & 41835 & -1777 \\
\hline 19 & 344658 & 1927405 & 177838 & 744577 & 22252 & -19583 \\
\hline 20 & 271164 & 2198569 & 206197 & 950775 & 28359 & 6107 \\
\hline 21 & 264860 & 2463429 & 217242 & 1168016 & 11044 & -17315 \\
\hline 22 & 259355 & 2722784 & 225337 & 1393353 & 8095 & -2949 \\
\hline 23 & 230485 & 2953269 & 231120 & 1624473 & 5783 & -2312 \\
\hline 24 & 257313 & 3210583 & 231012 & 1855485 & 108 & -5891 \\
\hline
\end{tabular}


Table 2. Working table, cont.

\begin{tabular}{|c|c|c|c|c|c|c|}
\hline $\begin{array}{l}\text { Week } \\
-1-\end{array}$ & $\begin{array}{c}\text { Sales } \\
-2-\end{array}$ & $\begin{array}{c}\text { Total Sales } \\
-3-\end{array}$ & $\begin{array}{c}\text { Exponential } \\
\text { smoothing } \\
-4-\end{array}$ & $\begin{array}{l}\text { Total } \\
-5-\end{array}$ & $\begin{array}{c}\Delta^{2} y \\
-6-\end{array}$ & $\begin{array}{l}\Delta^{3} y \\
-7-\end{array}$ \\
\hline 25 & 265185 & 3475767 & 235483 & 2090968 & 4471 & 4579 \\
\hline 26 & 214461 & 3690228 & 240532 & 2331501 & 5049 & 578 \\
\hline 27 & 233047 & 3923275 & 236100 & 2567601 & 4432 & -9481 \\
\hline 28 & 216963 & 4140238 & 235581 & 2803182 & 519 & 3913 \\
\hline 29 & 217461 & 4357699 & 232416 & 3035599 & $-\quad 3165$ & -2646 \\
\hline 30 & 219114 & 4576813 & 229874 & 3265472 & $\begin{array}{l}-\quad 2542 \\
\end{array}$ & 623 \\
\hline 31 & 203742 & 4780555 & 228045 & 3493517 & 1829 & 713 \\
\hline 32 & 187717 & 4968272 & 223913 & 3717430 & $-\quad 4131$ & -2302 \\
\hline 33 & 148933 & 5117205 & 217760 & 3935190 & 6153 & - 2022 \\
\hline 34 & 130007 & 5247212 & 206059 & 4141249 & - 11701 & -5547 \\
\hline 35 & 123898 & 5371110 & 193130 & 4334380 & -12929 & -1228 \\
\hline 36 & 98856 & 5469966 & 181361 & 4515741 & -11770 & 1159 \\
\hline 37 & 61598 & 5531564 & 167335 & 4683076 & -14026 & -2256 \\
\hline 38 & 42018 & 5573582 & 149360 & 4832435 & - 17975 & - 3949 \\
\hline 39 & 42823 & 5616404 & 131112 & 4963547 & -18248 & $\begin{array}{l}-\quad 273 \\
\end{array}$ \\
\hline 40 & 35153 & 5651558 & 116102 & 5079649 & $-\quad 15009$ & 3239 \\
\hline 41 & 38426 & 5689983 & 102341 & 5181990 & - 13761 & 1248 \\
\hline
\end{tabular}

Denote by $\mathrm{y}_{\mathrm{n}}$ consecutive values in column 5 , indexed by the week numbers from column 1 . The columns 6 and 7 show respectively the second and third differences of the numbers from column 5 . Let us recall that the second difference is defined by formula $\left(\Delta^{2} \mathrm{y}\right)_{\mathrm{n}}=\mathrm{y}_{\mathrm{n}}-2 \mathrm{y}_{\mathrm{n}-1}+\mathrm{y}_{\mathrm{n}-2}$ and the third one as $\left(\Delta^{3} \mathrm{y}\right)_{\mathrm{n}}=\mathrm{y}_{\mathrm{n}}-3 \mathrm{y}_{\mathrm{n}-1}+3 \mathrm{y}_{\mathrm{n}-2}-\mathrm{y}_{\mathrm{n}-3}$.

Note that the greatest second difference appears in the 17th week and corresponds to the number $y_{17}=411,153$ in column 5 .

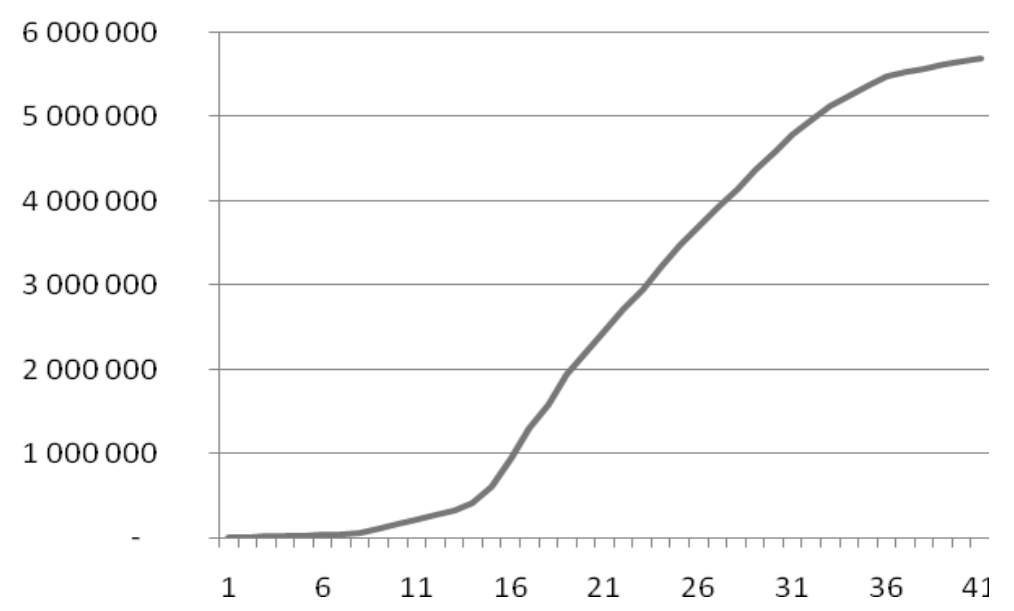

Figure 2. Total Sale of the bicycle accessories (data from column 3, Table 2) (source: own elaboration based on the collected data) 
Thus, using the final conclusions from the previous section we estimate the saturation level as:

$\mathrm{y}_{\max }=411,153 / 0.0729=5,639,961$.

Since the next second difference (calculated for the 18 th week) is only slightly less, that is, in 17 th week does not occur a distinct maximum, it seems that the above obtained value could be slightly enlarged.

We also see that the maximum of the third difference occurred in 10th week and corresponds to the value $\mathrm{y}_{10}=66,128$. Therefore the estimated saturation level will be equal to:

$\mathrm{y}_{\max }=66,128 / 0.0112=5,904,241$.

The both above estimates of the saturation level well correspond to the actual level resulting from the primary data (see Fig. 2).

Looking more broadly at the management, the Gompertz curve (or, for example, the logistic curve, the Bass function or others, depending on the specific data) could be a tool for an improvement of the communication between different departments of the company. The logistics department can define, for the purchases department, the time period, within which it must enforce the appropriate size of delivery from suppliers (purchasing department deals with setting delivery times in the company from which the data have been received), so that the logistics department would have sufficient time for the receipt and delivery of the goods to shops. The curve shows the managers of a particular store, when they have to prepare, for instance furniture, on which the seasonal goods will be exhibited (retail chain in the study used furniture for skis and ski accessories and summer furniture just for bikes and bike accessories). Properly prepared curves thus allow the departments to be better self-organized.

\section{Conclusions}

The smoothing constant 0.17 , used in the calculations of Table 2, has been selected empirically. We wanted to remove random fluctuations from the initial part of the time series. We have checked however, that one could take here any value in the range from approx. 0.1 to 0.2 .
We believe that our method, which consists of finding in a given time series, the points corresponding to zeroes of subsequent derivatives of the function describing the phenomenon under investigation, requires further study. One should examine its usefulness for others, possibly large datasets.

Furthermore, it seems that the method should be used together with existing methods of determining the characteristics of an S-shaped curve, for example, with the nonlinear least squares method.

\section{Bibliography}

[1] Graham R.L., Knuth D.E., Patashnik O. - Concrete Mathematics: A Foundation for Computer Science, Reading MA: Addison Wesley, 1994.

[2] Feng-Shang Wu, Wen-Lin Chu - Diffusion models of mobile telephony [in] Journal of Business Research, 63 (2010), pp. 497-501.

[3] Junseok Hwang, Youngsang Cho, Nguyen Viet Long - Investigation of factors affecting the diffusion of mobile telephone services: An empirical analysis for Vietnam [in] Telecommunications Policy 33 (2009), pp. 534-543.

[4] MahjanV., Muller E., and Bass F.M.. - New product diffusion models in marketing: A review and directions for research [in] Journal of Marketing, 54 (1990), pp. 1-26.

[5] Rządkowski G., Głażewska I., Sawińska K. Logistic function as a tool of planning [in] Foundations of Management 6 (2014), pp. 5770.

[6] Rządkowski G., Rządkowski W., Wójcicki P. On some connections between the Gompertz function and special numbers [in] Journal of Nonlinear Mathematical Physics 22 (2015), pp. 374-380.

[7] Stauffer D., Moss De Oliveira S., De Oliveira P.M.C., Sa Martins J.S. - Biology, Sociology, Geology by Computational Physisists, Monograph Series on Nonlinear Science and Complexity Vol. 1, Elsevier, 2006.

[8] Waliszewski P., Konarski J. - A Mystery of the Gompertz Function [in] Gabriele A. Losa (eds.), Fractals in Biology and Medicine, Birkhäuser Verlag 2005, pp. 277-286. 